[0212-7199 (2007) 24: 12; pp. 607-609] ANALES DE MEDICINA INTERNA Copyright (C) 2007 ARAN EDICIONES, S.L.

AN. MED. INTERNA (Madrid) Vol. 24, N. ${ }^{\circ} 12 ;$ pp. 607-609, 2007

\section{¿Qué papel ocupan los ARA-II en el tratamiento de la insuficiencia cardiaca? ¿Actuamos por evidencias/recomendaciones o por la presión de la industria farmacéutica?}

\author{
R. DE LA FUENTE CID, A. VARELA ROMÁN
}

Complexo Hospitalario Universitario. Santiago de Compostela. A Coruña

WHICH IS THE ROLE OF THE ANGIOTENSINE II RECEPTOR BLOCKERS (ARBS) FOR THE TREATMENT OF HEART FAILURE? DO WE WORK IN ORDER TO EVIDENCE/RECOMENDATIONS OR TO THE PHARMACEUTICAL INDUSTRY PRESSION?

\begin{abstract}
RESUMEN
La insuficiencia cardíaca crónica (ICC) representa la primera causa de ingresos hospitalarios en personas mayores de 60 años en nuestro medio. Además tanto su incidencia como su prevalencia continúan aumentando a pesar de nuevas estrategias terapéuticas.

Consideramos las recomendaciones de las guías de práctica clínica (GPC) un instrumento valioso a la hora de tomar decisiones, aunque en algunas ocasiones utilicen un lenguaje numérico poco claro. Tampoco debemos de olvidar que los pacientes que entran a formar parte de los ensayos clínicos en muchas ocasiones no son representativos de la realidad. Finalmente añadir que la industria farmacéutica, invierte grandes cantidades en investigación de principios activos, pero como empresa que es necesita obtener beneficios. Con todo ello, en este artículo intentamos analizar que papel ocupan los antagonistas del receptor de la angiotensina II (ARA-II) en la estrategia médica a la hora de planificar un tratamiento en el paciente con ICC.
\end{abstract}

PALABRAS CLAVE: Insuficiencia cardiaca crónica. Antagonistas del receptor de angiotensina.

\begin{abstract}
Chronic heart failure $(\mathrm{CHF})$ represents the first cause of hospitalitation in persons older than 60 years in our enviroment. Beside its incidence and prevalence is increasing although new therapeutic treatments. We think the recomendations of the guidelines of clinical practise are an important instrument to make decisions, although sometimes it is used a numeric language little clear. We do not have either to forget that sometimes patients selected for the clinical trials aren't really representative. Finally to add that pharmaceutical industry spends a lot of money on investigating active basics, but as company itself is needs to obtain benefits. With all of this, in this article we try to study the role of angiotensin II receptor blockers (ARBs) occuppy in the medic strategy for the planification of a patient's treatment with chronic heart failure.
\end{abstract}

KEY WORDS: Cronic heart failure. Angiotensin receptor blockers.

de la Fuente Cid R, Varela Román A. ¿Qué papel ocupan los ARA-II en el tratamiento de la insuficiencia cardiaca? ¿Actuamos por evidencias/recomendaciones o por la presión de la industria farmacéutica? An Med Interna (Madrid) 2007; 24: $607-609$.

\section{INTRODUCCIÓN}

No cabe duda de que el conocimiento y aplicación de las guías de práctica clínica, facilitan la toma de decisiones en el ejercicio de la medicina. También parece verdad aquello de que cada enfermo es diferente. Además hemos de considerar que los pacientes incluidos en los ensayos clínicos son muy seleccionados y con un seguimiento muy especial que pocas veces se parecen a los que tenemos sentados en nuestra consulta. Finalmente añadir, que en nuestra humilde opinión, el lenguaje utilizado para trasmitir las recomendaciones o los niveles de evidencia de las guías de práctica clínica (GPC) es expresado de forma poco clara. Veamos un ejemplo: la Sociedad Europea de Cardiología (ESC) hace una recomendación de clase IIa como aquella en que "el peso de la evidencia/opinión está a favor de la utilidad/eficacia”, y una recomendación clase IIb como aquella en la que "la utilidad/eficacia está menos indicada por la evidencia/opinión". No parece de mucha utilidad la información que nos trasmiten dichas recomendaciones a la hora de tomar una decisión.

A pesar de todas estas puntualizaciones, y siempre que tengamos la capacidad de extrapolar los datos de los ensayos clínicos para que nos proporcionen una orientación, creemos que las guías de practica clínica son un valioso instrumento en nuestro trabajo diario.

La introducción de los antagonistas del receptor de la angiotensina II (ARA-II) en el mundo de la insuficiencia cardíaca crónica (ICC) creó una gran expectación por su atractivo mecanismo de acción y por la menor incidencia de efectos adversos que los inhibidores del enzima convertidor de angiotensina (IECA). En los últimos años hemos asistido a un interés creciente en el posible papel de los ARA-II en la prevención de arritmias, y en la aparición de diabetes mellitus. Sin embargo, y aunque luego lo veremos con más detalle en los

Trabajo aceptado: 4 de octubre de 2007 
distintos estadios de la enfermedad, podemos adelantar de un modo general que no han sido capaces de superar a los IECA en la reducción en la morbilidad y mortalidad de estos pacientes. También debemos señalar que aunque a lo largo de todo el comentario hagamos referencia a los ARA-II, sería más correcto cuando tengamos que tomar una decisión terapéutica, hacerla con respecto a un fármaco determinado, pues a día de hoy no podemos hablar de un efecto de clase (1).

-Pacientes con disfunción sistólica ventricular izquierda asintomática. Tanto las guías de la ESC (2) como el American College of Cardiology (ACC)/ American Heart Association (AHH) (3) no recomiendan el uso de dichos fármacos en estos pacientes.

Ahora bien en el estudio OPTIMAAL (4) que comparó losartán con captopril en pacientes con disfunción sistólica ventricular izquierda después de sufrir un infarto agudo de miocardio (IAM), y en el estudio VALIANT (5) que comparó valsartán con captopril en pacientes con disfunción ventricular izquierda o insuficiencia cardíaca tras un IAM, se observó que la mortalidad era similar en ambos grupos y que la combinación de ambos fármacos no mejoraba las cifras de mortalidad.

En base a estos dos estudios y teniendo en cuenta que la mayoría de los pacientes que sufren un IAM no desarrollarán ICC, la ESC nos recomienda que en el IAM con signos de ICC o de disfunción ventricular sistólica izquierda los ARA-II y los IECA tienen efectos similares o equivalentes sobre la mortalidad. Sería una recomendación en la que hay evidencia (datos obtenidos de un solo ensayo clínico aleatorizado) y/o acuerdo general de que es un tratamiento beneficioso, útil y eficaz.

La ACC/AHA nos recomienda que se debe de administrar un ARA-II a los pacientes tras un IAM sin ICC que sean intolerantes a los IECA y tengan una fracción de eyección (FE) baja. Igualmente sería una recomendación en la que hay evidencia (datos obtenidos de un solo ensayo aleatorizado o de estudios no aleatorizados) y/o acuerdo general de que es una terapia beneficiosa, útil y eficaz.

-Pacientes con ICC con síntomas leves. En este grupo incluiríamos aquellos pacientes con una limitación ligera de la actividad física y en los que las actividades de la vida diaria producen disnea, fatiga o palpitaciones. Aparecerían síntomas en tareas que supongan un consumo superior a 7 METS en la escala de actividad específica de Goldman.

La ESC (1) recomienda que pueden utilizarse los ARA-II como una alternativa de los IECA en aquellos pacientes sintomáticos que presentan intolerancia a estos y conseguiremos de esta forma mejorar la morbilidad y mortalidad. Esta sería una recomendación en la que existe evidencia (datos obtenidos de un solo ensayo clínico aleatorizado) y/o acuerdo general en que es un tratamiento beneficioso, útil y eficaz.

Otra recomendación de la ESC para este grupo de pacientes es que los IECA y ARA-II tienen una eficacia similar sobre la mejoría de la morbilidad y mortalidad. Esta sería una recomendación en la que el peso de la evidencia (datos obtenidos de un solo ensayo clínico aleatorizado) y la opinión están a favor de su utilidad y eficacia.

Esto parece claro tras los resultados del estudio CHARM ALTERNATIVO (6) en el que pacientes con ICC sintomática y disfunción sistólica del VI (FE < 40\%) que no estaban tomando IECA por intolerancia, la introducción de ARA-II (candesartán) logró una reducción significativa tanto de la mortalidad como del número de hospitalizaciones por ICC.
La duda que se nos podía plantear en el papel de los ARAII en el tratamiento de la ICC era su uso combinado con IECA y betabloqueantes. En el estudio VAL-HEFT (7) comparó el efecto de añadir valsartán al tratamiento convencional en pacientes con ICC y FE $<40 \%$. La mayoría de los pacientes estaban recibiendo IECA (93\%) y betabloqueantes (35\%). La conclusión fue que al añadir valsartán se produjo una reducción significativa del objetivo combinado de morbi-mortalidad (muerte, hospitalización por IC o descompensación), pero la mortalidad fue similar en ambos grupos.

En el estudio CHARM AÑADIDO en el que comparó candesartán con placebo en pacientes con ICC sintomática por disfunción ventricular izquierda y en tratamiento con IECA. Los resultados mostraron que la adición del ARA-II se asociaba a una reducción significativa en el objetivo combinado de muerte y hospitalización por ICC, así como la reducción significativa de cada uno de dichos componentes por separado. Además un 55\% de los pacientes tomaba betabloqueantes y el beneficio de añadir candesartán fue similar entre los que tomaban betabloqueante y los que no (8).

Así pues la ESC (2) comunica que los ARA-II pueden utilizarse en combinación con los IECA en pacientes que permanezcan sintomáticos, para reducir la mortalidad, excepto en el caso post-infarto, como una recomendación en la que el peso de la evidencia (datos obtenidos de un ensayo clínico aleatorizado) y la opinión está a favor de su utilidad y eficacia. Y además dicha asociación se recomienda con la misma fuerza para reducir las hospitalizaciones por ICC pero con un nivel de evidencia mayor (datos obtenidos de múltiples ensayos clínicos aleatorizados o meta-análisis).

La ACC/AHA (3) en este sentido recomienda que puede considerarse la asociación de un ARA-II a pacientes con sintomatología persistente y con FE disminuida, que están siendo tratados con terapia convencional.

Con respecto a que los ARA-II representen una alternativa a los IECA en caso de intolerancia a estos, la ACC/AHA (3) y sin nivel de evidencia, recomienda que puede considerarse a los ARA-II como alternativa para pacientes que hayan desarrollado angioedema cuando tomaban IECA, pero hay un pequeño grupo de pacientes que también pueden desarrollar angioedema con los ARA-II, por lo que se aconseja una precaución máxima al indicar ARA-II.

- Pacientes con ICC con síntomas severos. Pertenecerían a este grupo incluiríamos aquellos pacientes que presentan una limitación moderada-importante de la actividad física diaria, pues esta ya le produce disnea, fatiga o palpitaciones, aunque no en reposo. Las actividades que requieren más de 5 METS en la escala de Goldman, producirían síntomas. También deberíamos incluir en este grupo aquellos pacientes con incapacidad para realizar ninguna actividad física sin limitación, ya que la sintomatología puede estar presente incluso en reposo, aunque este grupo probablemente debería ser considerado diferente y precisaría medidas especiales de la ICC refractaria.

La ESC (2) establece la misma recomendación y con el mismo nivel de evidencia que en los pacientes con síntomas leves, por la cual debe añadirse un ARA-II a pesar de diurético, IECA y betabloqueante, siempre que el paciente permanezca sintomático. Pero esta misma guía, tras lo observado en el estudio RALES (9) en el que demostró que la adición de espironolactona presentaba un beneficio en la mortalidad, no deja claro cual es el siguiente fármaco que se debería de aña- 
dir, un ARA-II o un antagonista de la aldosterona (AA). Dado que ningún ensayo a comparado ARA-II con AA en esta situación, tendremos la posibilidad de elegir cual es el tercer fármaco que se debe añadir.

Por su parte la ACC/AHA (3) igualmente hace las mismas recomendaciones que en pacientes con síntomas leves, pero en lo referente a que fármaco añadir a la terapia convencional si continúan sintomáticos no establece unas recomendaciones claras. Únicamente establece que no tiene utilidad/eficacia y en algunos casos puede ser dañino, la utilización de cuadruple terapia, con un nivel de evidencia basada en opinión de expertos y/o pequeños estudios retrospectivos o registros (10).

-Pacientes con ICC con fracción de eyección conservada. En contraste con los numerosos ensayos clínicos en ICC con disfunción sistólica, en este tipo de pacientes existen pocos estudios, por lo que no existen unas recomendaciones firmes de la ESC (2) ni de la AHA/ACC (3). Es probable que en estos casos debamos olvidarnos de las guías y pautar un "tratamiento a la carta" para cada paciente, por supuesto encaminado a aumentar la supervivencia, conseguir una mejoría sintomática mediante la tolerancia al ejercicio y reducir el número de reingresos hospitalarios.

Si parecen claras unas directrices establecidas a la hora de programar el tratamiento: empleo de fármacos bradicardizantes para aumentar el tiempo de llenado ventricular, intentar mantener el ritmo sinusal por la importancia de la contracción auricu-

\section{Bibliografía}

1. Tamargo J, Caballero R, Gómez R, Núñez L, Vaquero M, Delpón E, Características farmacológicas de los ARA-II ¿Son todos iguales? Rev Esp Cardiol 2006; 6: 4C-9C.

2. Swedberg K, Cleland J, Dargie H, Drexler H, Follath F, Komajda M, et al; Task Froce for the Diagnosis and Treatment of Chronic Heart Failure of the European Society of Cardiology: Guidelines for the diagnosis and treatment of chronic heart failure. Eur Heart J 2005; 26: 1115-40.

3. Hunt SA, Abraham WT, Chin MH, Feldman AM, Francis Gs, Ganiats TG et al; American college of Cardiology; American Heart Association Task Force on Practice Guidelines; American College of Chest Pyisiciam; International Society for Heart and Lung Transplantation Heart Rhythm Society. ACC/AHA 2005 Guideline Update for the Diagnosis and Management of Chronic Heart Failure in the Adult. Circulation. 2005; 112: 154-235.

4. Dickstein K, Kjekshhus J. Effects of losartan and captopril on mortality and morbidty in high_risk patients after acute myocardial infartion: the OPTIMAAL randomised trial. Optimal trial in Myocardial Infartion with Angiotensin II Antagonist Losartan. Lancet 2002; 360: 752-60.

5. Pfeffer MA, McMurray JJ, Velázquez EJ, Rouleau JL, Kober L, Maggioni AP, et al. Valsartan, Captopril, or both in myocardial infarction complicated by heart failure, left ventricular dysfunction, or both. N Engl J Med 2003; 249: 1893-906.

6. Granger CB, McMurray JJV, Yusuf S, Held P,Michelson EL, Olofsson $\mathrm{B}$, et al. For the CHARM Investigators and Committees. Effects of candesartan in patients with chronic heart failure and reduced left-ventricular systolic function intolerant to angiotensin-converting-enzyme inhibitors: the CHARMAN-Alternative trial Lancet 2003; 362: 772-6.

7. Cohn JN, Tognoni G. A randomized trial of the angiotensin-receptor bloc- lar en esta situación de alteración de elasticidad y/o distensibilidad ventricular, control de la presión arterial (PA) con un valor recomendable inferior a $130 / 80 \mathrm{~mm} \mathrm{Hg}$, y por supuesto el empleo de fármacos destinados a reducir la hipertrofia ventricular izquierda (HVI) y la fibrosis miocárdica (11).

Y que nos dicen las evidencias a este respecto; en el estudio LIFE en el que se comparó losartán frente a atenolol en pacientes hipertensos con hipertrofia ventricular izquierda, se demostró que el primero producía una mayor reducción de la hipertrofia ventricular, una mayor reducción de eventos cardiovasculares y una menor incidencia de nuevos diagnósticos de diabetes mellitus (12).

En otro pequeño estudio (21 pacientes) Warner JG demostró que en pacientes hipertensos con ICC con función sistólica conservada, la utilización de losartán aumentaba de forma significativa el tiempo de ejercicio y mejoraba la calidad de vida empleando el código Minnesota (13).

Pero sin lugar a dudas el mayor ensayo clínico en ICC es el programa CHARM con sus tres brazos. Si nos fijamos concretamente en la rama de función sistólica conservada (CHARM PRESERVADO) con 3025 pacientes seguidos durante 36 meses los resultados finales no demuestran beneficio significativo en cuanto a mortalidad, aunque si en el número de hospitalizaciones, tasa de ICTUS o infartos de miocardio y en la tasa de nuevos diagnósticos de diabetes mellitus (14). ker valsartan in chronic heart failure. N. Engl Med 2001; 345: 1667-75.

8. McMurray JJ, Ostergren J, Swedberg K, Granger CB, Held P, Michelson EL, et al. effects of candesartan in patients with chronic heart failure and reduced left-ventricular systolic function intolerant to angiotensin-converting-enzyme inhibitors: the CHARM-Added trial. Lancet 2003; 362: 367-771.

9. Pitt B, Zannad F, Remme WJ, Cody R, Castaigne A, Perez A, et al. The effect of spironolactone on morbidity and mortality in patients with severe heart failure. Randomized Aldactone Evaluation Study Investigators. N Engl J Med 1999; 341: 709-17.

10. Cleland JGF, Clark AL. Delivering the cumulative benefits of triple therapy for heart failure. Too many cooks will spoil the broth. J Am Coll Cardiol. 2003; 42: 1226-33.

11. Grigorian L, Varela A, Virgos A, Rigueiro P, García JM, González-Juanatey JR. Evolución a largo plazo de la prescripción de fármacos en pacientes hospitalizados por insuficiencia cardíaca congestiva. Influencia del patrón de disfunción. Rev Esp Cardiol 2005; 58: 381-88.

12. Dahlof B, Devereux RB, Kjeldsen SE, et al. Cardiovascular morbidity and mortality in the Losartan Intervention For Endpoint in hypertensión study (LIFE): A randomised trial against atenolol. Lancet 2002; 359: 995-1003

13. Warner JG Jr, Metzger DC, Kitzman DW, et al. Losartan improves exercise tolerance in patients with diastolic dysfunction and a hypertensive response to exercice. J Am Coll Cardiol 1999; 33: 1567-72.

14. Yusuf S, Pfeffer MA, Ewedbergk, et al for the CHARM Investigators and Committees. Effects of candersantan in patients with chronic heart failure and preserved left-ventricula ejection fraction: the CHARM-Preserved Trial. The CHARM-Overall programme. Lancet 2003; 362: 777-81. 